\title{
Preliminary characterization of jejunocyte and colonocyte cell lines isolated by enzymatic digestion from adult and young cattle ${ }^{\text {is }}$
}

\author{
Suzanne Loret ${ }^{\mathrm{a}, 1}$, Dorina Rusu ${ }^{\mathrm{a}, 2}$, Benaissa El Moualij ${ }^{\mathrm{b}}$, Bernard Taminiau ${ }^{\mathrm{c}}$, Ernst Heinen ${ }^{\mathrm{b}}$, \\ Guy Dandrifosse ${ }^{\mathrm{a}}$, Jacques Mainil ${ }^{\mathrm{c}, *}$ \\ a Laboratory of Biochemistry and General Physiology, Faculty of Medicine, University of Liège, Campus du Sart Tilman, B-4000 Liège, Belgium \\ ${ }^{\mathrm{b}}$ Department of Human Histology, Centre of Immunology, CRPP, Faculty of Medicine, University of Liège, Campus du Sart Tilman, B-4000 Liège, Belgium \\ ' Bacteriology, Department of Infectious Diseases, Faculty of Veterinary Medicine, University of Liège, Campus du Sart Tilman, B-4000 Liège, Belgium
}

\section{A R T I C L E I N F O}

Article history:

Accepted 3 December 2008

\section{Keywords:}

Jejunocytes

Colonocytes

Cattle

Transformation

SV40 LT

\begin{abstract}
A B S T R A C T
In the present study we developed an enzymatic approach (through the use of collagenase and dispase) to isolate bovine intestinal epithelial cells. Using this method, freshly isolated jejunocytes could be distinguished from simultaneously isolated colonocytes, as the jejunocytes specifically exhibited the small intestinal peptidase gene transcript, as well as an active alkaline phosphatase. The transformation of both types of cell suspension was performed by retroviral infection, using reproduction-defective viruses bearing the gene coding for the large T antigen of the leukaemia simian virus (SV40). The success of the transfection was demonstrated by (1) a significant increase in cell passage numbers (52-53 vs. 7 passages for nontransfected cells), (2) the detection of both the large $T$ transcript and the large $T$ antigen in transformed cells. Possible contamination and progressive substitution of bovine primocultures by non-bovine lineages available in the laboratory was excluded, as the transformed cells presented a bovine typical karyotype. Most transfected cells kept an epithelial morphology after transformation. They also maintained the expression of FABP and enterocyte specific enzymes (brush-border associated maltase and IAP). However, levels of specific activity of these enzymes were low, suggesting that cell differentiation is not completely achieved under the applied culture conditions.
\end{abstract}

(c) 2008 Elsevier Ltd. All rights reserved.

\section{Introduction}

Interacting in different ways with enterocytes is one of the properties of microbial enteric and invasive pathogens that will cause a perversion of enterocyte physiological functions and that can lead, directly or indirectly, to the production of diarrhoea and/or to the invasion of the enterocyte. In addition, the intestinal epithelium can represent a portal of entry for microbial toxins (mycotoxins, botulinum toxin f.i.) and drugs (antibiotics f.i.) (Didierlaurent et al., 2006; Holland, 1990; Mayer, 2003). Study of these interactions at the cellular and molecular levels in the natural hosts is most often impossible for ethical and financial reasons, and experimental results are not always transposable from laboratory animal species, due to the existence of host-specific mechanisms. In addition, the

\footnotetext{
is S.L. and D.R. participated equally in this study whose G.D. was the head promoter.

* Corresponding author. Tel.: +32 4 3669522; fax: +32 43664261.

E-mail address: jg.mainil@ulg.ac.be (J. Mainil).

1 Present address: Department of Biology, University of Namur (FUNDP), Rue de Bruxelles, 61, B-5000 Namur, Belgium.

2 Present address: Diagenode sa, Avenue de l'Hôpital, 1, Tour GIGA (3ème étage), B-4000 Liège, Belgium.
}

age of the animal and the intestinal segment can be important in assessing the receptivity of the host (Chong et al., 2007; Chu and Walker, 1993; Hashim et al., 2004; Mundy et al., 2007; Santos and Baümer, 2004). Amongst the more user-friendly approaches, primary or immortal cells in culture are particularly favoured today as a first step in studying such interactions (McCormick, 2003).

The use of primary cell cultures is limited due to the high rate of cell mortality following the dissociation of the intestinal epithelium, the very high incidence of culture contamination inherent in the persistence of the intestinal flora and the variability of cell culture properties due to inter-individual variability between samples. Moreover, primary enterocytes rarely multiply for more than 10 generations, which restricts the number of experiments per cell lot and consequently the comparative value of the results (Bader et al., 2000; Dimier-Poisson et al., 2004; Dopido et al., 2004; Evans et al., 1994; Macartney et al., 2000).

Immortal enterocyte cell lines have been derived from human colon adenocarcinomas (Caco-2, HT-29, T84) (Murakami and Masui, 1980; Rousset et al., 1979; Zweibaum et al., 1985) and from rat and chick embryos after spontaneous transformation (Quaroni et al., 1979; Velge et al., 2002). Intestinal continuous cell lines have also been obtained from different intestinal segments of humans, pigs, mice, and rats, after conditional immortalization by oncogene 
transfection (Berry et al., 1988; Brandsch et al., 1998; Emami et al., 1988, 1989; Hosoya et al., 2004; Jat et al., 1991; Kaeffer et al., 1993; Quaroni and Beaulieu, 1997; Tabuchi et al., 2000, 2002; Tavelin et al., 1999). The tumour large $T$ antigen of the simian leukaemia virus 40 (SV40 LT) is the oncogene most frequently used to immortalize cells by transfection (Jha et al., 1998; Toouli et al., 2002).

The aim of this study was to obtain conditionally immortalized bovine jejunocytes and colonocytes isolated from one adult bovine and one young calf. The cells were derived following the adaptation of an enzymatic digestion approach (Dibb-Fuller et al., 2001; Föllmann et al., 2000) coupled to a mechanical dissection, which had been previously successful in yielding primocultures of both jejunocytes and colonocytes from adult cattle (Rusu et al., 2005). Immortalization was achieved by transfection with the tumour large T antigen of the simian leukaemia virus 40 (SV40 LT). Differentiation of the immortalized cells was studied under different growth conditions.

\section{Materials and methods}

\subsection{Primary cell isolation and culture}

Jejunocytes and colonocytes were isolated from proximal jejunum and spiral colon segments from one adult bovine sampled at a local slaughterhouse, as previously described (Rusu et al., 2005). The two digestion steps with collagenase (300 U/ml; Sigma) and dispase $\left(0.1 \mathrm{mg} / \mathrm{ml}\right.$; Gibco/BRL) were performed at $37^{\circ} \mathrm{C}$ in a shaking bath for 15 and $45 \mathrm{~min}$ (jejunum) or for 20 and $60 \mathrm{~min}$ (colon). The digested epithelia were subsequently scraped from the mucosa. The preparation was depleted of lymphocytes and fibroblasts by 5 isopicnic centrifugations in Dubelcco's modified Eagle medium (D-MEM) containing $2 \%$ sorbitol (Sigma) and by decanting into a culture flask devoid of any coating, to favour the adherence of residual fibroblasts (Rusu et al., 2005). The epithelial cell enriched suspension was cultured at $37{ }^{\circ} \mathrm{C}$ in a humidified incubator (Heraeus Instruments) and a $5 \% \mathrm{CO}_{2}$ atmosphere, in culture flasks coated with type I collagen (Roche Diagnostics), to allow adherence of the epithelial cells. Jejunocytes and colonocytes were also isolated by enzymatic digestion from proximal jejunum and spiral colon segments of one 36-h-old calf after euthanasia, with the following adaptations because of the greater fragility of the intestinal mucosa at that age: only one digestion step of $30 \mathrm{~min}$ (jejunum) or $45 \mathrm{~min}$ (colon) and a reduced collagenase concentration (150 U/ml; Sigma).

The culture medium of the primary enterocytes isolated from the adult bovine was D-MEM with antibiotics, antimycotics, bovine foetus calf serum and growth factor supplements (Rusu et al., 2005). The culture medium of the primary enterocytes isolated from the young calf was Episerf (Gibco/BRL). Since it contains no foetal calf serum, Episerf medium was supplemented with $0.2 \%$ bovine pituitary extract (Gibco/BRL), 1\% of an insulin-transferrinselenium solution (Gibco/BRL), $10 \mathrm{nM}$ hydrocortisone (Sigma), $20 \mathrm{nM}$ triiodothyronine (Sigma), $10 \mathrm{ng} / \mathrm{ml}$ Epidermal Growth Factor (Sigma), $10 \mu \mathrm{g} / \mathrm{ml}$ linoleic acid/Albumin (Sigma), 1\% Glutamax (Gibco/BRL), 1\% Non Essential Amino-Acids (NEAA, Gibco/BRL), $1 \%$ sodium pyruvate (Sigma) and $1 \%$ antibiotic-antimycotic solution (Gibco/BRL). The culture medium was changed every 2 or 3 days. Confluent cells were subcultured at a split ratio of $1: 2$, were frozen in liquid nitrogen and were stored at $1.5 \times 10^{6}$ cells per vial, in $1 \mathrm{ml}$ of D-MEM containing 10\% DMSO (Merck) and 10\% FBS (Hyclone Perbio Sciences) until further use.

\subsection{Viability and morphology studies}

The viability of the freshly isolated enterocytes was estimated by staining their nuclei with a mixture of ethidium bromide (Sigma) and acridin orange (Sigma) in PBS (Rusu et al., 2005). Using this process, the nuclei of living cells stain green while the nuclei of dead cells stain red. The morphology of the transformed cell lines was studied by electron microscopy (JEOL JEM-100CX microscope at $80 \mathrm{kV})$.

\subsection{Cell transformation and culture}

The bovine enterocytes were transfected with an origin minus temperature sensitive mutant of a retrovirus vector coding for the SV40 LT immortalizing oncogene (O'Hare et al., 2001; Stamps et al., 1994). The constructs were received from Profs. O'Hare and Jat from LICR (London, UK) and the retroviral suspension was released by amphotrophic virus producer cell lines (or packaging cells) kindly provided by the same laboratory (O'Hare et al., 2001). Briefly, PA317, a second-generation mouse amphotropic packaging cell line was used to produce the retroviruses expressing SV40 U19tsA58 LT antigen (temperature sensitive SV40 large T antigen). PA317 cells were cultured in D-MEM (Gibco/BRL) supplemented with $1 \%$ antibiotic-antimycotic solution, 10\% FBS (Hyclone), and L-glutamine to obtain a $6 \mathrm{mM}$ final concentration in the medium. The viral suspension used for primoculture infection, was the cell medium collected $16 \mathrm{~h}$ after cell confluence. Prior to addition to primoculture, this medium was centrifuged at $140 \mathrm{~g}$ for $2 \mathrm{~min}$, in order to discard detached packaging cells, and was then filtered through a sterile nylon membrane (pore diameter: $0.45 \mu \mathrm{m}$; Fischer Bioblock). The culture of retrovirus packaging cells and the transfection were accomplished in a level 3 biosafety containment facility of the Centre for Research on the Prion Protein (CRPP, University Hospital, University of Liège, Belgium) to ensure there was no accidental release or exposure.

After thawing, the cells were suspended in their respective culture medium and grown in type I collagen coated flasks in the same conditions as described above, till 50-75\% confluence (i.e. in an actively multiplying phase). The supernatants were replaced by the retroviral suspension (i.e. the packaging cell culture medium) for $18 \mathrm{~h}$ (=infection stage) in the presence of polybrene $(8 \mu \mathrm{g} / \mathrm{ml}$; Sigma) in order to obtain a mild and temporary permeability of the cell membrane, allowing the entry of the immortalizing material into the target cells. The viral suspension was then discarded and replaced by fresh culture medium to stop incubation of the infection. Cells were allowed to grow until they reached confluence. The cells isolated from the calf were submitted to two infection stages. The cells in which the immortalizing gene had become inserted into the genome were selected by growth at $33^{\circ} \mathrm{C}$ in the culture medium for the SV40 LT transfection in the presence of geneticin G418 (750 $\mu \mathrm{g} / \mathrm{ml}$; Gibco/BRL) for seven days (=selection stage). Once the transfected cells were confirmed to be negative for retrovirus production, they were transferred to a biosafety level 2 laboratory for routine subculture.

In the case of the calf cells, at the end of the selection process performed in 6-well plates, resulting clusters with epithelial morphology were delineated with a marker at the bottom of the plate. The culture medium was then removed by aspiration and the cell layer was washed once with PBS. The rings of cloning were firmly glued onto the culture dish surface with the aid of sterile silicone grease (Merck). Cells present in delineated rings were detached by trypsin and EDTA treatment (Gibco/BRL, $30 \mu \mathrm{l}$ per ring) for a period of $3 \mathrm{~min}$ and subsequently removed with the upper ends of sterilized $1 \mathrm{ml}$ pipette tips (Greiner Bio-One). Recovered cell suspensions were transferred into a wheel of 24-well plates, containing $1 \mathrm{ml}$ of culture medium, which was changed after $6 \mathrm{~h}$. Cell cloning was performed by flow cytometry using a FACS Vantage SE cell sorter and the CloneCyt/CellQuest software (Becton Dikinson) to obtain one cell per well of a 96 -well microtitre plate. Several of the acquired clones were spread in culture and six of 
them were chosen for further study. Their longevity was first estimated by techniques routinely used to determined cell proliferation. Cells were seeded in 24-well plates at the ratio of 20,000 cells per well. They were grown in supplemented Episerf medium for one week. Each day, cells from 3 wells were detached using $100 \mu \mathrm{l}$ of trypsin/EDTA solution and were counted. Among those six calf transfected cell clones, two (one from jejunum and one from colon) were chosen for further characterization.

The medium of the routine culture of transfected enterocytes (from the adult bovine or from the calf) were the same as the ones described for primocultures (see Section 2.1). Confluent cells were subcultured at a split ratio of $1: 2$ in order to estimate their longevity.

\subsection{Expression assays}

Enterocyte culture mRNAs were analysed by RT-PCR for the transcription of the genes coding for SV40 LT antigen, villin, Fatty Acid Binding Protein (FABP), Zona Occludens 1 (ZO1), vimentin, E-cadherin, and Small Intestinal Peptidase (SIP). Quality controls of mRNA extraction and cDNA reverse-transcription were performed targeting the expression of GlycerAldehyde-3-Phosphate DeHydrogenase (GAPDH). All primers and PCR conditions have already been described (Rusu et al., 2005).

\subsection{Karyotype analysis}

The transfected enterocytes were grown to $50-70 \%$ confluence and incubated for $1 \mathrm{~h}$ at $37^{\circ} \mathrm{C}$ in their usual supplemented medium with $0.1 \mu \mathrm{g} / \mathrm{ml}$ colcemide (Gibco/BRL). They were harvested in PBS and centrifuged at $500 \mathrm{~g}$ for $5 \mathrm{~min}$. The pellet was resuspended in $10 \mathrm{ml}$ of a hypotonic $\mathrm{KCl}$ solution ( $0.075 \mathrm{M}$; Gibco/BRL) and incubated for $40 \mathrm{~min}$ at $37^{\circ} \mathrm{C}$. After the addition of $3 \mathrm{ml}$ of a methanol-acetic acid solution $(3: 1 \mathrm{v}: \mathrm{v})$ to fix the cells, they were centrifuged for $5 \mathrm{~min}$ at $500 \mathrm{~g}$ and resuspended for $15 \mathrm{~min}$ in $10 \mathrm{ml}$ of the same solution. This step was repeated twice before the pellet was resuspended in $3 \mathrm{ml}$ of the same solution. One drop was transferred onto a microscope glass slide and allowed to air dry. The preparation was subsequently stained with a solution of quinacrine dihydrochloride in milliQ water $(5 \mathrm{~g} / \mathrm{l}$; Merck) for $5 \mathrm{~min}$, washed in tap water and observed under fluorescent microscope.

\subsection{Cell immunostaining assays}

Several monoclonal antibodies were used to characterize the transfected enterocytes by direct immunostaining or Western blot, namely: anti-pan-cytokeratin (mixtures of clones C-11, PCK-26, CY-90, KS1A3, M20, A53, and B/A2; Sigma), anti-E-cadherin (clone 34; Becton Dickinson Biosciences), anti- $\alpha$ smooth muscle actin (clone 1A4; Sigma), anti-vimentin (clone V9; Sigma) and anti-cytokeratin peptide 18 (clone KS-BA2; Sigma). 3T3-fibroblast cultures were used as positive controls for the vimentin and $\alpha$-actin staining and Caco- 2 cells were used as a positive control for the cytokeratin staining. The efficacy and stability of the SV40 LT transfection were studied every 10th passage by the detection of the LT antigen, and also by Western blot, using a monoclonal anti-SV40 LT antibody received from Prof. P. Jat (Jat et al., 1991; LICR, London, UK).

Twenty micrograms of proteins extracted from the transformed enterocytes after sonication were separated by electrophoresis in SDS-PAGE (10\%) and transferred onto nitrocellulose membranes (Biotrade PVDF Transfer Membrane, $0.45 \mu \mathrm{m}$; Pall Corporation, Life Science). The membranes were sequentially incubated for $1 \mathrm{~h}$ at 37 ${ }^{\circ} \mathrm{C}$ in the blocking solution (PBS supplemented with Tween 20 [Sigma P1379; 0.1\% v:v] and with powdered milk [Nestle; 5\% v:v]), in the blocking solution containing the primary antibody (diluted
$1: 1000)$ and in the blocking solution containing the secondary antibody conjugated to peroxidase (an anti-mouse IgG diluted 1:1000; Sigma). After three washings in PBS, positive reactions were revealed by the addition of peroxidase substrate, as already described (Rusu et al., 2005).

\subsection{Enzymatic activities}

Primary and transfected enterocytes were enzymatically typed by measuring the levels of activity of Intestinal Alkaline Phosphatase (IAP), which is associated in vivo with both jejunocytes and colonocytes, and the levels of MALtase (MAL), a brush-border disaccharidase essentially associated in vivo with jejunocytes (Dahlqvist, 1964). Enzymatic activity was measured in triplicate on 3-day post-confluent cells at different passages using spectrophotometric methods after cell disruption by sonication (30 s on ice; Branson Sonic Power Company), as described earlier (Rusu et al., 2005). Enzymatic activity was reported to the protein content (or specific forms of enzymatic activity expressed as $\mu \mathrm{mol} / \mathrm{min} / \mu \mathrm{g}$ of proteins), assessed according to the method of Bradford (1976).

\section{Results}

\subsection{Cell isolation and characterization}

Jejunocytes and colonocytes were successfully isolated by enzymatic digestion from proximal jejunum and spiral colon segments of one adult bovine and of one 36-h-old calf. In agreement with previous results (Rusu et al., 2005), all cell primocultures were consistent with epithelial cell and enterocyte identity regarding the morphology and the expression of specific markers, the Fatty Acid Binding Protein (FABP) and the villin (VIL). Jejunocytes from adult intestinal fragments, but not colonocytes, were also positive for the transcription of the gene coding for the small intestine peptidase (SIP; Fig. 1). Similar results for FABP and VIL were obtained for jejunocytes and colonocytes from a young calf, but those cells were not tested for SIP.

\subsection{Cell transformation}

Following the first passage, all four bovine enterocyte primocultures were successfully submitted to immortalizing transfec-

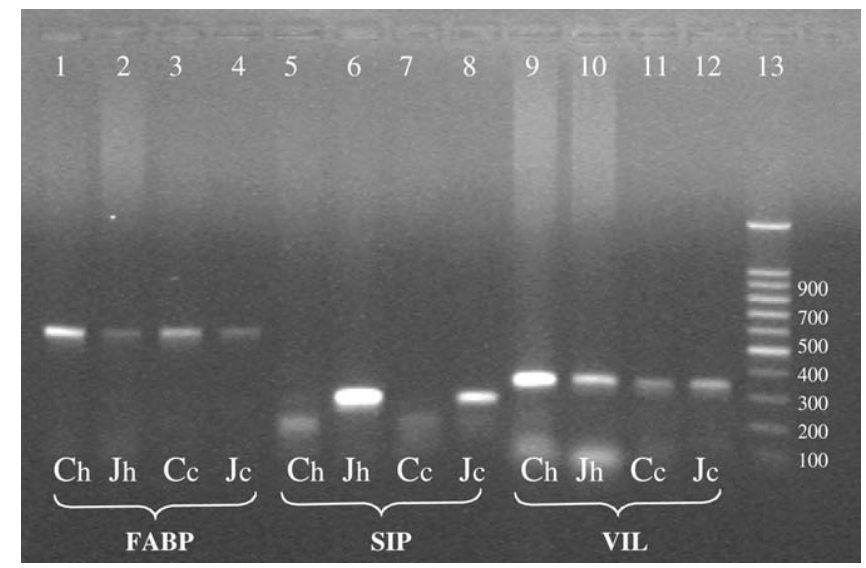

Fig. 1. RT-PCR analysis of transcripts of FABP (lanes 1-4), SIP (lanes 5-8) and VIL (lanes 9-12) in bovine intestinal homogenates of colon ( $\mathrm{Ch}$ ) and jejunum (Jh) compared to homogenates of bovine enterocytes isolated from colon $(\mathrm{Cc})$ and jejunum (Jc) of one adult bovine. The fragment size (bp) of amplified cDNA fragments was determined, using a standard mix ladder (lane 13), after migration on agarose $(1 \%)$ gels and subsequent staining with ethidium bromide. 
tion using a retroviral suspension bearing the SV40 large (L) T antigen construct. Indeed, compared to the longevity of untreated primocultures (7 passages), the number of cell generations observed after cell transfection with transforming retrovirus was markedly increased to about 50 passages after 300 days of continuous subculture process (Fig. 2). The transformation resulted in four transformed cell lines of adult bovine jejunocytes (J8 and $\mathrm{J} 8 / 13$ ) and colonocytes (C8 and C8/13) and in two transformed cell clones of calf jejunocytes (J8A2) and colonocytes (C8B1). Though these calf transfected enterocytes are actual cell clones, they will be named "cell lines", like the adult bovine transfected enterocytes, for the sake of clarity of the manuscript. All transformed lines had closely similar growth rates. The efficacy of the SV40 LT transfection was confirmed in all six cell lines by Western blot (Fig. 3) and by RT-PCR with specific primers (Fig. 4). In agreement with the thermosensitive expression of the LT antigen construct, the band intensity was slightly higher at $33.5^{\circ} \mathrm{C}$ than at $37{ }^{\circ} \mathrm{C}$ or $39{ }^{\circ} \mathrm{C}$, although no quantitative assessment of the amount of protein was performed.

\subsection{Transformed enterocyte characterization}

The karyotypes (Fig. 5) of the six cell lines revealed the typical chromosomal markers of the bovine species and the absence of any human or murine cell chromosomal markers. All six cell lines presented features of epithelial cells reported earlier for primocultures, namely a typical pavement-like aspect and the expression of cytokeratine 18 (Fig. 6a and b). However, all the six cell lines synthesized the vimentin protein (perfect overlapping of vimentin and cytokeratin staining in Fig. 6a and d), a signature of connective tissue, though they did not express the $\alpha$ actin, another marker of mesenchymal cells (there was no overlapping of cytokeratin or vimentin with $\alpha$ actin staining in Fig. 6b and e or in Fig. $6 c$ and f).

Electron micrographs showed that all the six cell lines formed only a few not well-shaped microvilli at the apical pole (Fig. 7) and that they were deficient in any actual layer of microvilli (or brush border). Moreover, transcripts expected in polarized epithelial cells, like those for villin and E-cadherin, were not detected in transformed confluent cells (data not shown). Also, the transcript
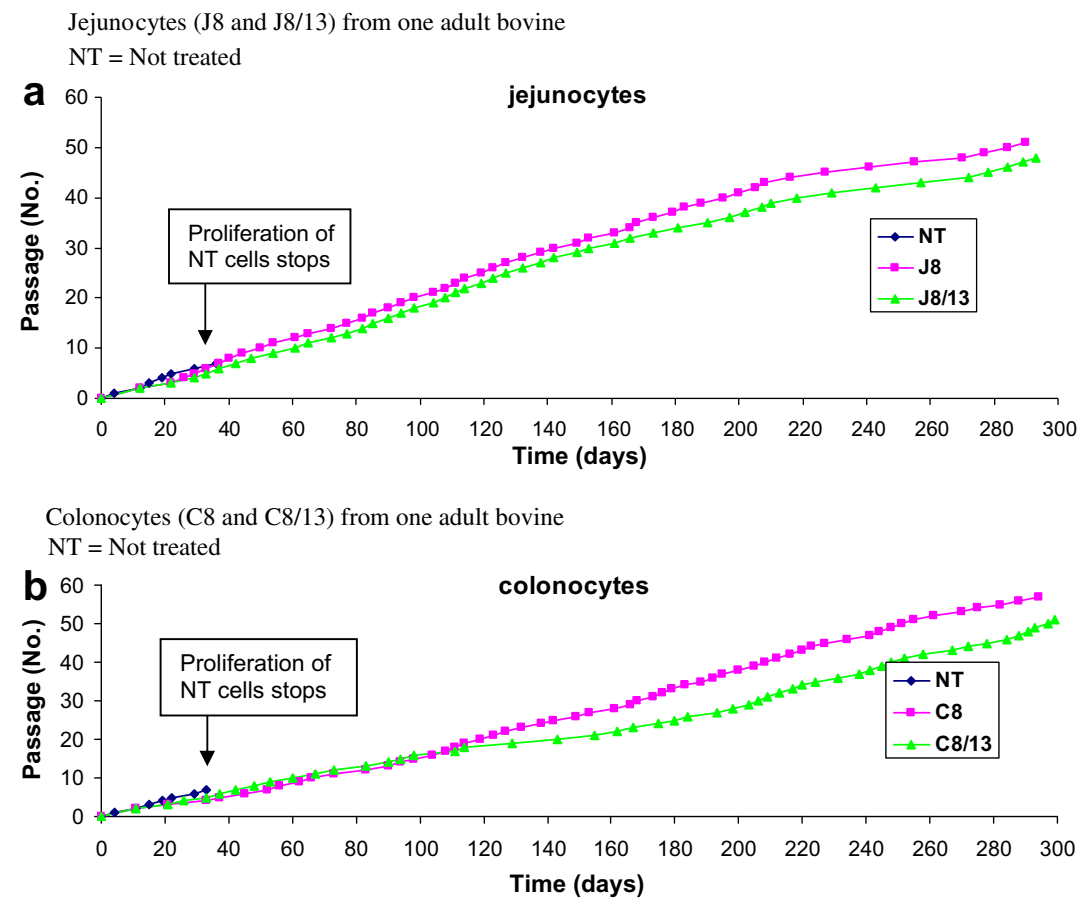

$$
\begin{aligned}
& \text { Jejunocytes (J8/A2) and colonocytes (C8B1) from one 36-h old calf } \\
& \text { JNT = untreated jejunocytes; CNT = untreated colonocytes }
\end{aligned}
$$

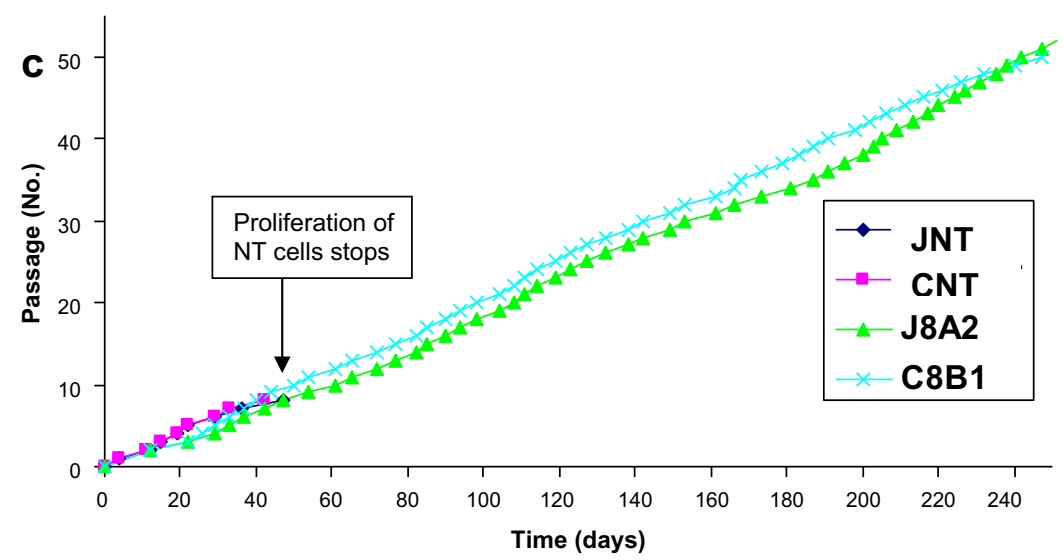

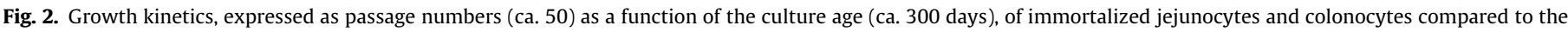
untreated primoculture (NT) isolated from (a, b) one adult bovine and (c) one 36-h-old calf. 
a

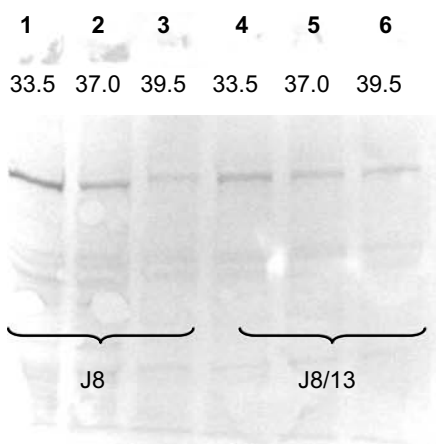

7

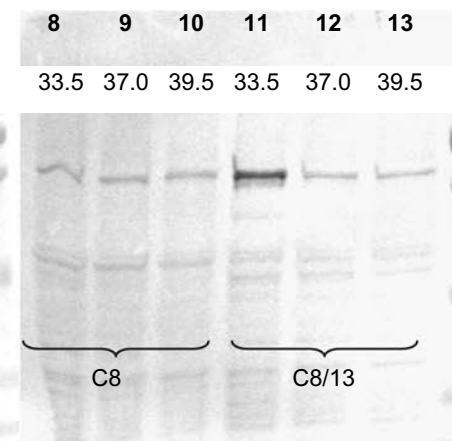

b

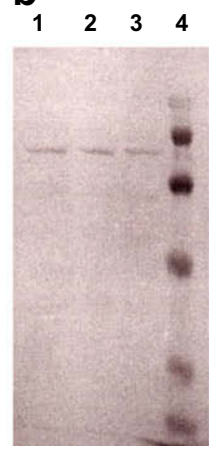

Fig. 3. (a) Western blot of SV40 large T antigen in immortalized enterocytes from one adult bovine intestinal epithelium. Lanes 1-6: immortalized jejunocyte lines (J8 and J8/ 13); lanes 8-13: immortalized colonocyte lines (C8 and C8/13); lane 15: packaging cells producing the retroviral suspension used to transform enterocytes primocultures. For each line, the immunodetection was performed on cells grown at optimal "proliferation" temperature (i.e. $33.5^{\circ} \mathrm{C}$ : lanes $1-4-8-11$ ), "physiologic" temperature (i.e. $37.0^{\circ} \mathrm{C}$ : lanes 2-5-9-12) and optimal "differentiation" temperature (i.e. $39.5^{\circ} \mathrm{C}$ : lanes $3-6-10-13$ ). The size of the detected antigen was determined using a broad range protein standards molecular weight ranging from 5.7 to $198 \mathrm{KDa}$ (lanes 7 and 14). (b) Western blot of SV40 large T antigen in immortalized enterocytes from one 36-h-old calf intestinal epithelium. Lane 1: packaging cells; lane 2: immortalized jejunocyte line (J8A2); lane 3: immortalized colonocyte line (C8B1); lane 4: broad range protein standards molecular weight ranging from 5.7 to $198 \mathrm{kDa}$.

a

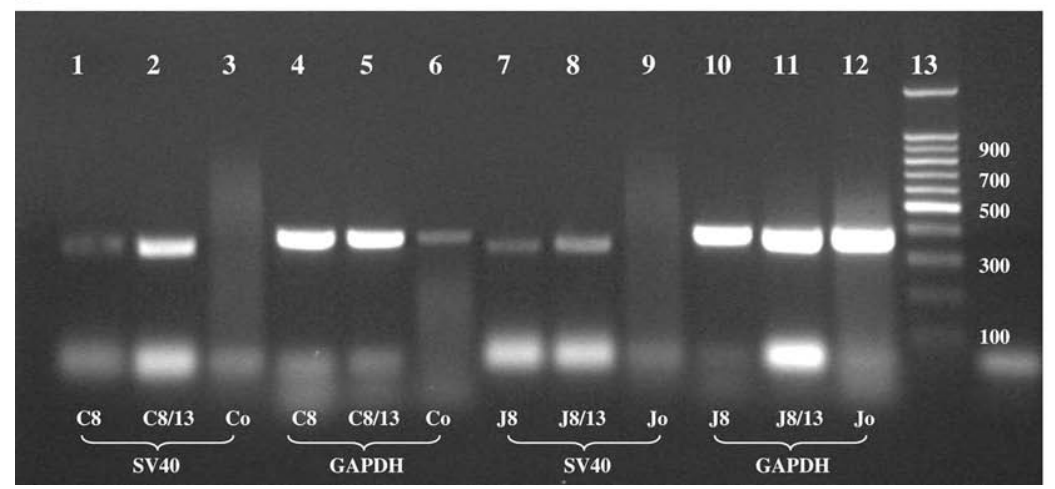

b

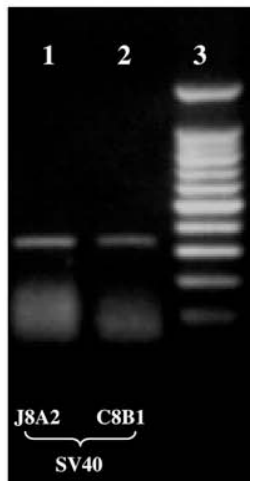

Fig. 4. (a) Detection of the transcription of the gene coding for the SV40 LT antigen by RT-PCR in immortalized enterocytes from one adult bovine intestinal epithelium. Lanes 1-2: immortalized colonocyte lines (C8 and C8/13) compared to freshly isolated colonocytes (or C0: lane 3). Lanes 7-8: immortalized jejunocyte lines (J8 and J8/13), compared to freshly isolated jejunocytes (J0: lane 9). Analysis of GAPDH transcription in all samples served as internal quality control of mRNA isolation and cDNA reversetranscription (lanes 4-6 corresponding to colonocyte samples and lanes 10-12 corresponding to jejunocyte samples). (b) Detection of the transcription of the gene coding for the SV40 LT antigen by RT-PCR in immortalized enterocytes from one 36-h-old calf intestinal epithelium. Lane 1: jejunocyte line (J8A2). Lane 2: colonocyte line (C8B1). The fragment size (bp) of amplified cDNA was determined, using a standard mix ladder (lane 13 in a and lane 3 in b), after migration on agarose (1\%) gels and subsequent staining with ethidium bromide.

a

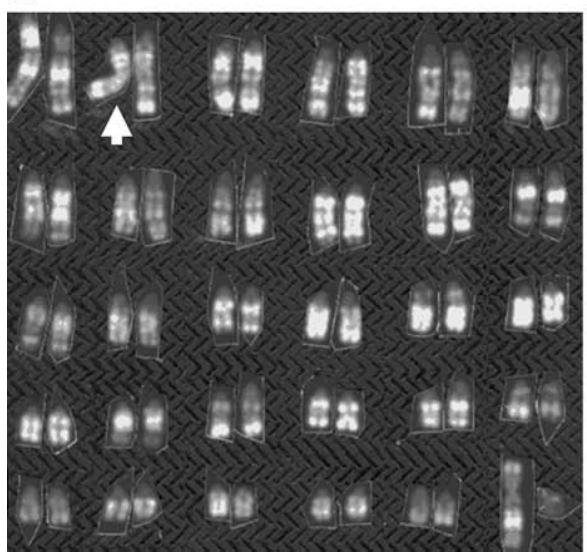

b

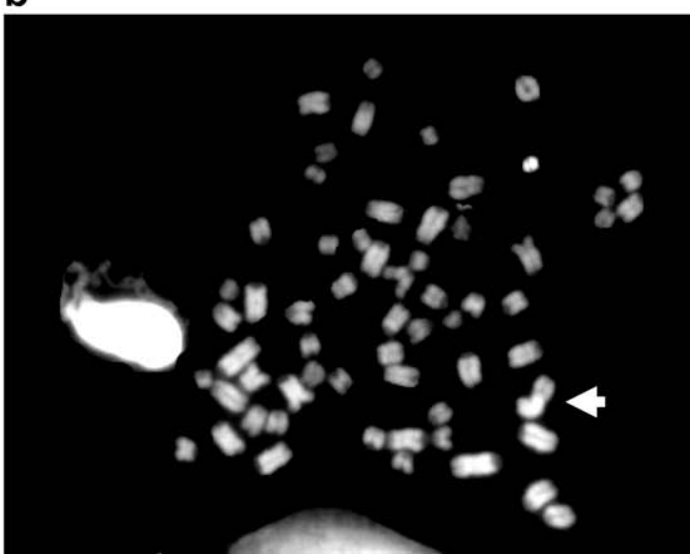

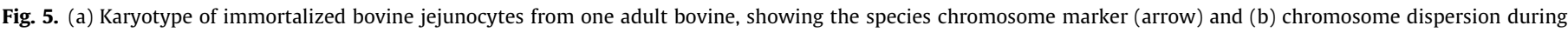
mitosis, showing the species chromosome marker (arrow). 

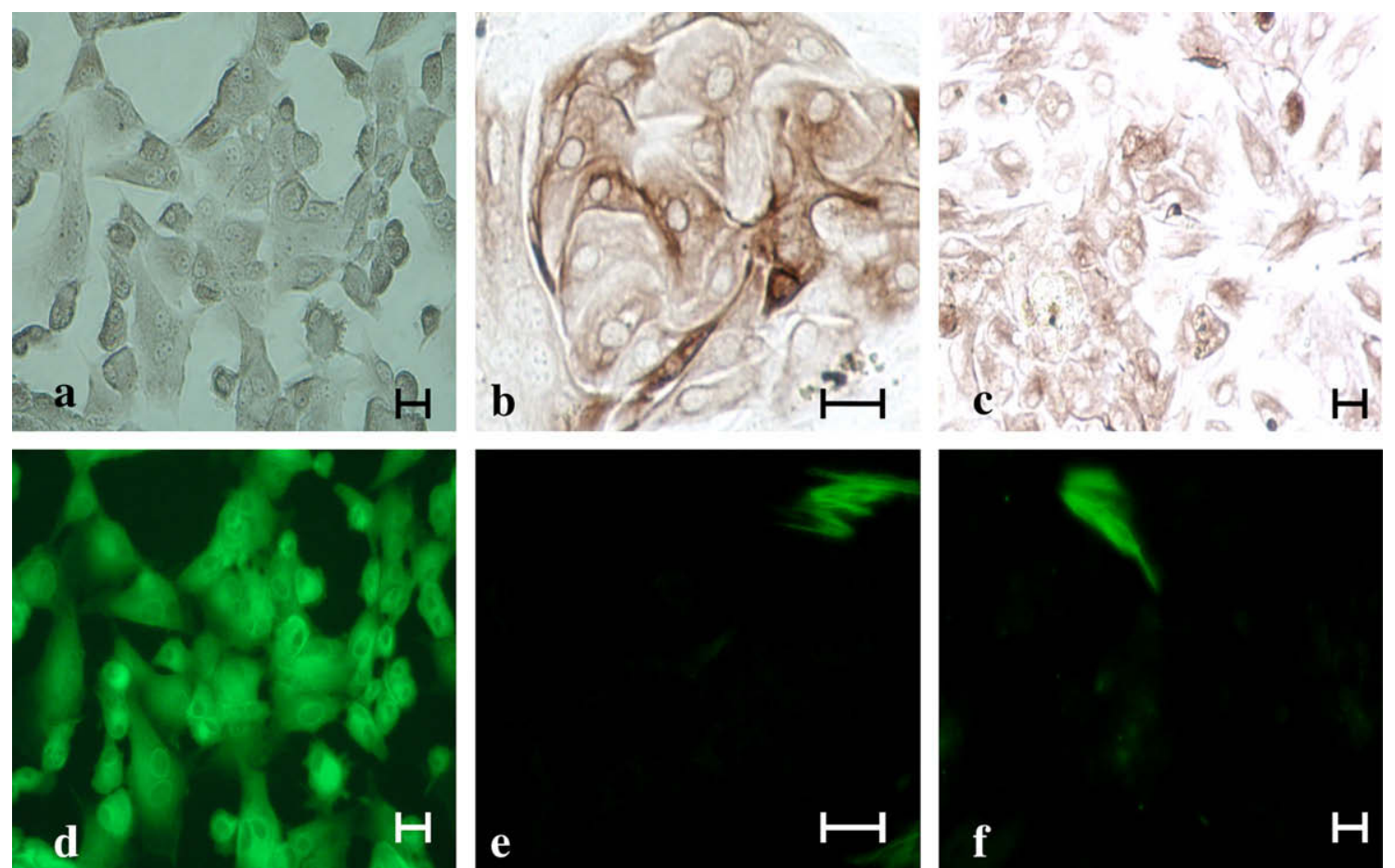

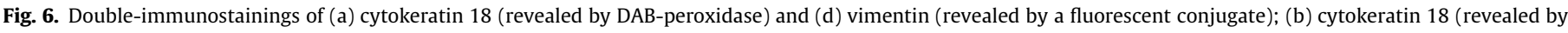

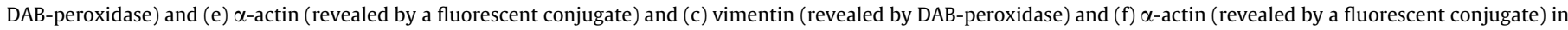
cultures of bovine enterocytes. Barr: $15 \mu \mathrm{m}$.
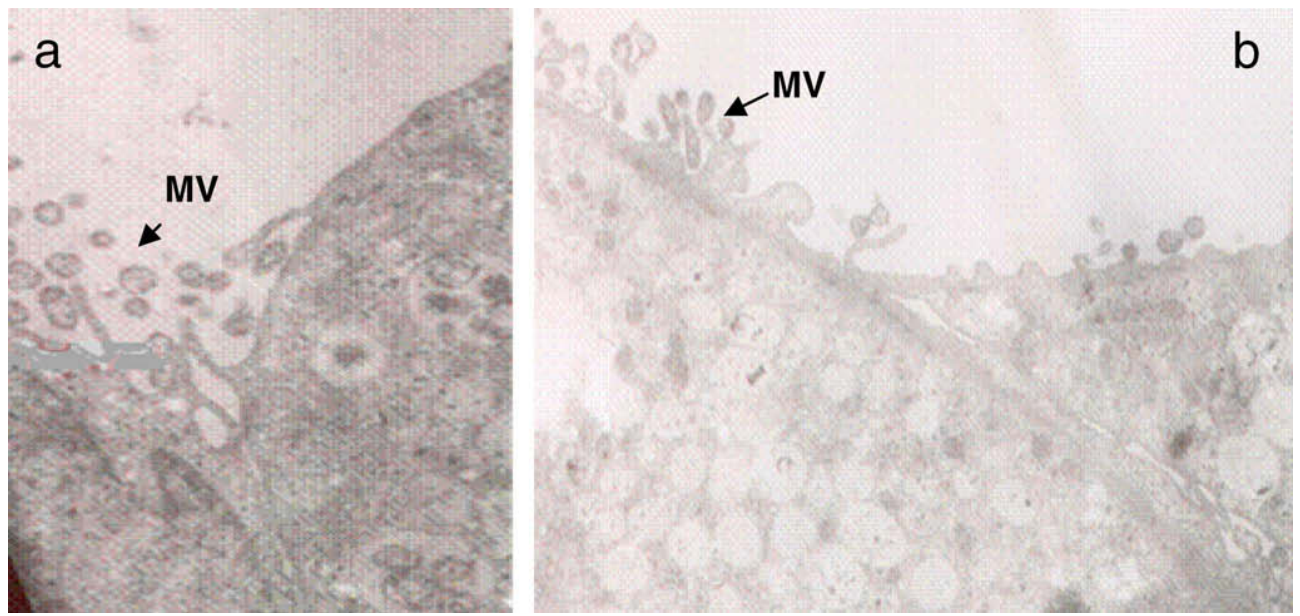

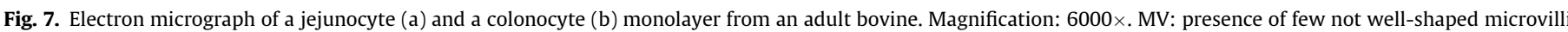
(MV).

for the jejunocyte specific marker, SIP, was not detected in the transformed jejunocytes from the adult bovine (Fig. 8), though this gene was clearly transcribed in primocultures (Fig. 1). By contrast, the expression of the FABP-encoding gene estimated by RT-PCR remained stable after transformation throughout the passages when compared to primocultures (Fig. 8), confirming the enterocyte identity of the six transformed cell lines.

The levels of activity of IAP and of MAL were measured at several culture passages (Fig. 9a and b). The MAL-specific activity remained low throughout the passages and was similar in jejunocytes and colonocytes (Fig. 9b). In the first passages following the transformation of enterocytes from the adult bovine intestine segments (see passages 10 to 20 in Fig. 9a), IAP was typically a marker of jejunocytes. However, as the number of passages increased, the IAP activ- ity tended to decrease in both jejunocyte lines. Surprisingly, IAP activity also became detectable in colonocytes. Similarly, jejunocytes obtained from the young calf showed a slightly higher level of IAP activity than calf colonocytes (Fig. 9c). However, this IAP activity was far lower than in the jejunocytes of adult origin.

\section{Discussion}

The lack of bovine immortal intestinal cell models impairs the study of the molecular basis of the host-specific interactions between enterocytes and microbial enteric or invasive pathogens, microbial toxins or drugs. Indeed, results obtained with other cell models cannot always be fully transposed to enterocytes (Bai 
a

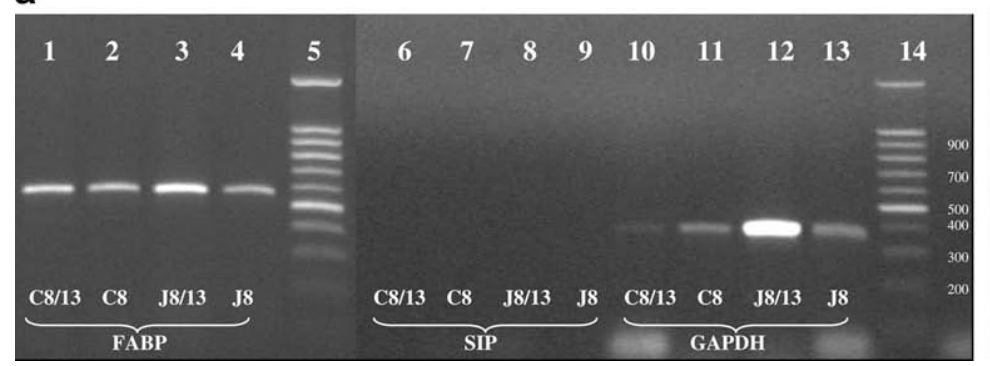

b

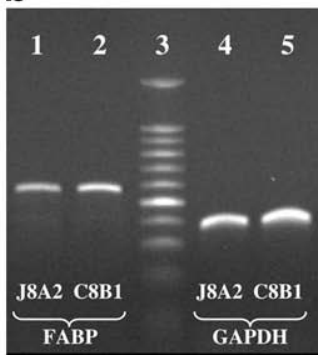

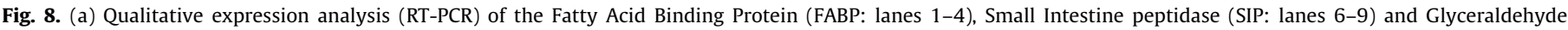

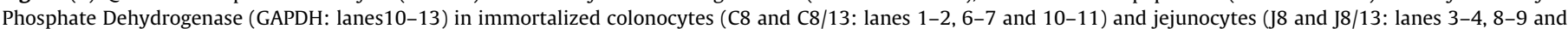

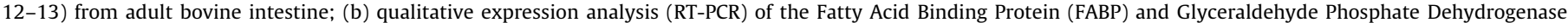

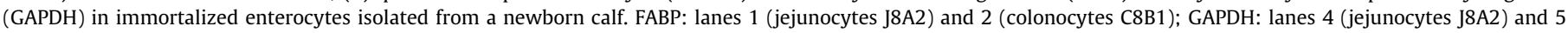

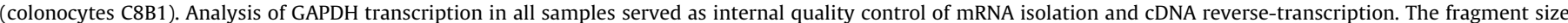

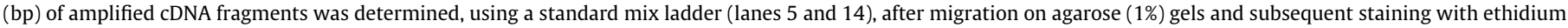
bromide.
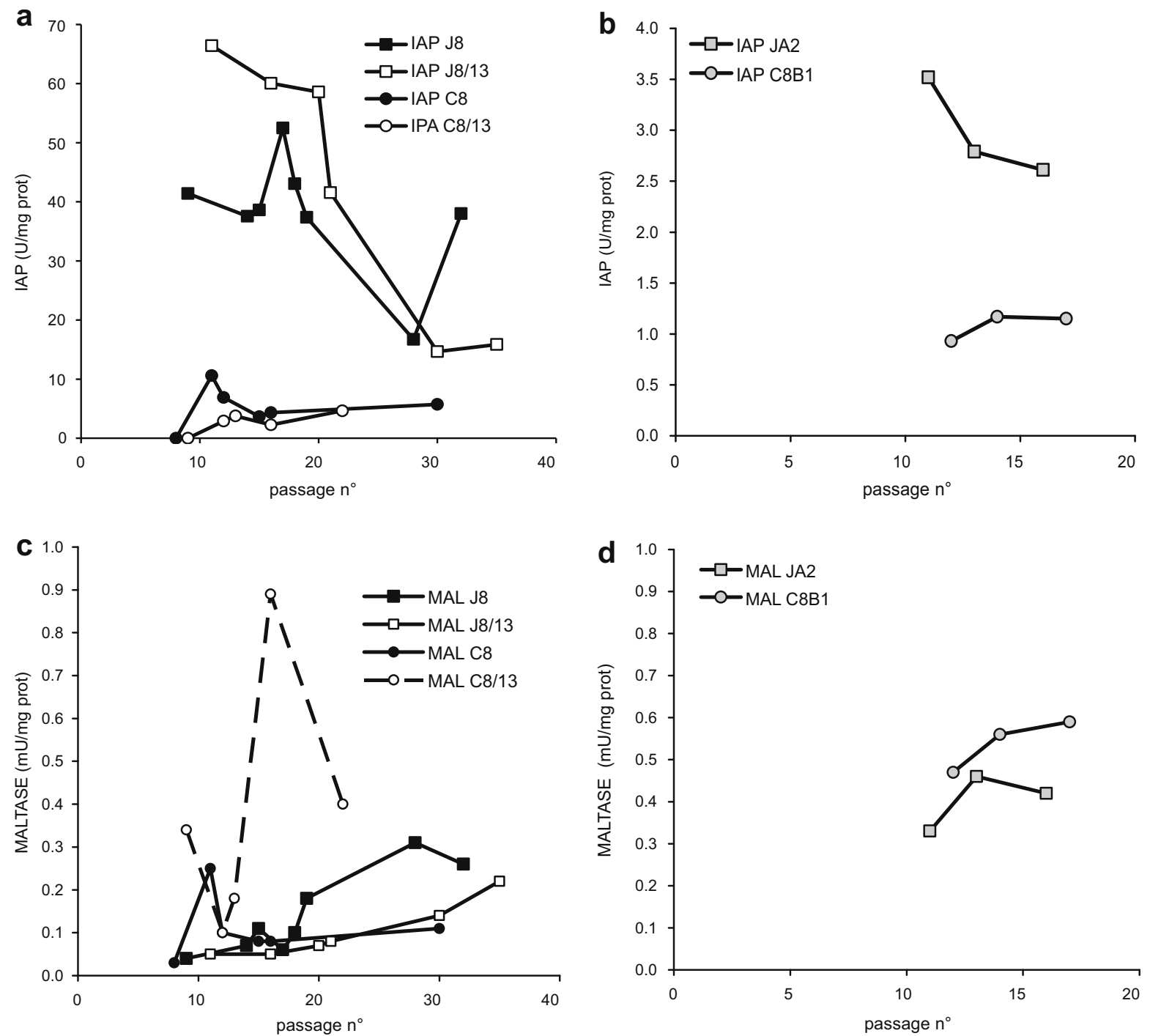

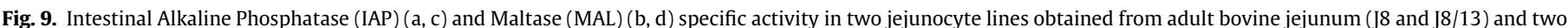

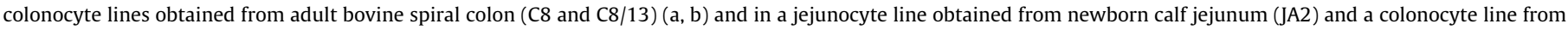
newborn calf spiral colon (C8B1) (c, d). Activity values were mean values from enzymatic assays performed in triplicate on two samples for each tested passage.

et al., 2008; Schuller et al., 2007). Conditional immortalization of primocultures of jejunocytes and colonocytes from an adult bovine and a young calf was performed by transfection of the large $\mathrm{T}$ anti- gen of the simian leukaemia virus 40 (SV40 LT) after cell isolation by enzymatic digestion and mechanical dissection of the intestinal mucosa. Six lines of transformed adult and young jejunocytes (J8, 
$\mathrm{J} 8 / 13$ and J8A2) and colonocytes (C8, C8/13 and C8B1) were obtained by retroviral transformation. The SV40 LT transfection was confirmed by growth in the presence of geneticin, by an increase in the number of cell generations (over 50 for ca. 10 months vs. 6-7 for primocultures) and by the detection of the expression of the LT antigen by RT-PCR and Western blotting.

The identification of the six transformed cell lines to bovine enterocytes relies upon their epithelial aspect under optic and electron microscopy and upon the results of the tests performed on them and/or on the original primocultures: typical bovine karyotype, transcription of typical epithelial and enterocyte genes (coding for FABP, SIP, villin), the presence of typical epithelial and enterocyte cytoskeleton and structural markers (cytokeratin 18, Ecadherin). Though FABP is not a $100 \%$ enterocyte specific marker (being also expressed in hepatocytes), contamination of isolated enterocytes by hepatocytes is unlikely and FABP can therefore be considered as a specific enterocyte marker in the present context. Interestingly, jejunocytes freshly isolated from the adult bovine, but not transformed jejunocytes, expressed SIP-encoding gene.

The uniformization of the feature characteristics of the enterocytes in culture over cell generations strongly suggests a progressive dedifferentiation of the four primary jejunocyte and colonocyte cell lines: similar growth curves and a pavement-like aspect of the monolayers, similar morphology under electron microscopy with only a few and not well-shaped microvilli, similar low (though variable between lines) levels of activity of IAP and MAL in comparison with freshly isolated intestinal mucosa, and the absence of transcription of the SIP-encoding gene in transformed jejunocytes. A likely explanation for this absence of full differentiation could reside in the selection during the primocultures of lowly differentiated stem cells, which can multiply better than they can fully differentiate in vitro, as already discussed (Rusu et al., 2005). In the case of the calf jejunocytes, however, the low level of IAP activity compared to the adult jejunocytes is in agreement with the known immature state of newborn intestinal epithelium (Lebenthal and Lebenthal, 1999).

Transformed cells also expressed the gene coding for vimentin, a marker of mesenchymal cells. Therefore, vimentin expression could represent a cell culture contamination with fibroblasts. However, the lack of $\alpha$ actin expression, another typical marker of fibroblasts cells, strongly suggests that the transformed cells were not of mesenchymal origin. Moreover, synthesis of vimentin in primary and transformed enterocytes has already been reported (Kaeffer et al., 1993; Rusu et al., 2005) and there are exceptions to its association with mesenchymal cells (Hunt and Davis, 1990; Lelouard et al., 1999; Moll et al., 1982; Onori et al., 2001). The presence of vimentin in enterocytes could actually be attributed to a posttranscriptional derepression of its synthesis in primocultures following the dissociation of the epithelium layer, as suggested elsewhere (Rusu et al., 2005). In agreement with this hypothesis, one class of enterocytes, the M cells, found in the intestinal epithelium covering the Peyer's patches has been found constitutively to express the vimentin protein (Lelouard et al., 1999; Onori et al., 2001). The fact that it is possible for $M$ cells to be obtained in vitro from enterocytes by a lymphocyte-induced phenotypic conversion (Kerneis et al., 1997) suggests a link between the production of vimentin and the brush-border effacement of the progenitor enterocyte. Accordingly, the present results suggest that production of vimentin could be associated with a similar dedifferentiation process during the immortalization of the enterocytes. Further studies would need to focus on verifying the reversal process i.e. the silencing of vimentin expression in correlation with enterocyte differentiation, particularly the formation of microvilli and of a brush border.

In reference to the undifferentiated state of carcinoma enterocyte cultures obtained by others (Wice et al., 1985; Zweibaum et al., 1985), different culture conditions were tested to increase the degree of differentiation of the four bovine enterocyte lines obtained in the present study (data not shown). For instance, sodium butyrate, a microbial metabolite that promotes Caco-2 cell differentiation (Abbott, 2004; Levy et al., 2003; Pryde et al., 2002) had only a poor effect on differentiation of the transformed bovine enterocytes in vitro, just as previously observed in primocultures (Rusu et al., 2005). Also the depletion of glucose in culture medium, a condition known to stimulate differentiation of human enterocytes in vitro was clearly not successful. Nor was the attempt to grow the cells at $39^{\circ} \mathrm{C}$, a temperature normally inhibiting of cell proliferation induced by LT stable transfection, to the benefit of cell differentiation. Moreover, cells adhered and formed poorly polarized monolayers on microporous inserts, known to trigger Caco-2 cell differentiation (Halleux and Schneider, 1991). Consequently, the more promising approaches are those favouring the reexpression of key genes involved in differentiation, such as E-cadherin, which was silent under the experimental conditions used in the present study. Integrin and E-cadherin are indeed key components in the enterocyte differentiation process. This is because integrindependent adhesion of the extracellular matrix is known to induce the polarization of Caco- 2 cells through the reinforcement of Ecadherin-actin complexes, which in turn operates on the abundance of the hepatic nuclear factor 4a (Sauvaget et al., 2002; Peignon et al., 2006). It would therefore be interesting to restore the stable expression of E-cadherin in transformed bovine cells, following the transfection strategies developed earlier by Beaulieu's team (Escaffit et al., 2005). However, the forced expression of Ecadherin alone appeared to not be sufficient to trigger significantly the differentiation process in HIEC cells (Escaffit et al., 2005). So, the second step towards activation of the E-cadherin-induced differentiation pathway would be to culture E-cadherin-forced expressing cells on surfaces coated with chimeric E-cadherin Fc homophilic ligand (Peignon et al., 2006), a method that proved to be efficient in inducing differentiation in vitro. Finally, as E-cadherin activity is also affected by $\mathrm{Cdx}$ homoedomain transcription factors (Cdx1 and Cdx2) (Domon-Dell et al., 2002; Qualtrough et al., 2002; Keller et al., 2004), a strategy leading to an increased expression of those factors in transformed bovine enterocytes should contribute to their differentiation in vitro. Further studies would also need to include the characterization of cell cultures without antibiotics, as these substances are know to sometimes affect significantly the growth, the morphology and the functions of cell cultures (Kuhlmann, 1996).

The transformation of adult and young jejunocytes and colonocytes from cattle with the SV40 LT antigen was successful, opening up perspectives in the study of the interactions of not only host-, but also age- and segment-specific, pathogens and molecules, which interact with the intestinal epithelium, once their actual differentiation state is precisely defined and fully restored.

\section{Acknowledgements}

The authors are grateful to Dr. C. Herens of the Department of Cytogenetics of the University of Liège for performing the study of karyotypes and to Profs. P. Jat and M. O'Hare (Ludwig Institute for Cancer Research, London, UK) (LICR-University College, London) for kindly providing the packaging cells producing the retrovirus and the anti-LT antigen monoclonal antibody. The authors are also grateful to Mrs. M. Klimek for the enzymatic activity measurements and to Mrs. N. Decloux for the sample preparation for electron microscopy. The authors also thank Dr. O. Peulen for his critical reading of the manuscript. This study was supported by a grant from the Federal Public Service, Public Health, Security of the Food Chain and Environment (S-6046 and S-6138). 


\section{References}

Abbott, A., 2004. Microbiology: gut reaction. Nature 22, 284-286.

Bader, A., Hansen, T., Kirchner, G., Allmeling, C., Haverich, A., Borlak, J.T., 2000 Primary porcine enterocytes and hepatocyte cultures to study drug oxidation reactions. British Journal of Pharmacology 129, 331-342.

Bai, L., Schüller, S., Whale, A., Mousnier, A., Marches, O., Wang, L., Ooka, T. Heuschkel, R., Torrente, F., Kaper, J.B., Gomes, T.A., Xu, J., Phillips, A.D., Frankel, G., 2008. Enteropathogenic Escherichia coli 0125:H6 triggers attaching and effacing lesions on human intestinal biopsy specimens independently of Nck and TccP/TccP2. Infection and Immunity 76, 361-368.

Berry, R.D., Powell, S.C., Paraskeva, C., 1988. In vitro culture of human foetal colonic epithelial cells and their transformation with origin minus SV40 DNA. British Journal of Cancer 57, 287-289.

Bradford, O.M.M., 1976. A rapid and sensitive method for the quantitation of microgram quantities of protein utilizing the principle of protein-dye binding. Analytical Biochemistry 72, 248-254

Brandsch, C., Friedl, P., Lange, K., Richter, T., Mothes, T., 1998. Primary culture and transfection of epithelial cells of human small intestine. Scandinavian Journal of Gastroenterology 33, 833-838.

Chong, Y., Fitzhenry, R., Heuschkel, R., Torrente, F., Frankel, G., Phillips, A.D., 2007. Human intestinal tissue tropism in Escherichia coli 0157:H7-Initial colonization of terminal ileum and Peyer's patches and minimal colonic adhesion ex vivo. Microbiology 153, 794-802.

Chu, S.H., Walker, W.A., 1993. Bacterial toxin interaction with the developing intestine. Gastroenterology 104, 916-925.

Dahlqvist, A., 1964. Method for assay of intestinal disaccharidases. Analytical Biochemistry 7, 18-25.

Dibb-Fuller, M.P., Best, A., Stag, D.A., Cooley, W.A., Woodward, M.J., 2001. An in vitro model for studying the interaction of Escherichia coli 0157:H7 and other enteropathogens with bovine primary cell cultures. Journal of Medical Microbiology 50, 759-769.

Didierlaurent, A., Simonet, M., Sirard, J.C., 2006. Innate and acquired plasticity of the intestinal immune system. Cellular and Molecular Life Science 62, 12851287.

Dimier-Poisson, T.H., Bout, D.T., Quiéré, P., 2004. Chicken primary enterocytes: inhibition of Elmeria tenella after activation with crude interferon-gamma supernatants. Avian Disease 48, 617-624.

Domon-Dell, C., Wang, Q., Kim, S., Kedinger, M., Evers, B.M., Freund, J.M., 2002 Stimulation of the intestinal CDX2 homeobox gene by butyrate in colon cance cells. Gut 50, 525-529.

Dopido, R., Rodriguez, C., Gomez, T., Alosta, N.G., Diaz, M., 2004. Isolation and characterization of enterocytes along the intestinal tract of gilhead seabream (Sparus aurata L.). Comparative Biochemistry and Physiology. Part A. Molecular and Integrative Physiology 132, 21-31.

Emami, S., Mir, L., Chanut, P., Chastre, E., Chatelet, F., Feunteun, J., Gespach, C., 1988 Expression of histamine and vasoactive intestinal peptide (VIP) receptors in immortalized rat fetal intestinal cells. Agents and Actions 23, 276279.

Emami, S., Mir, L., Gespach, C., Rosselin, G., 1989. Transfection of fetal rat intestinal epithelial cells by viral oncogenes: establishment and characterization of the E1A-immortalized SLC-11 cell line. Proceedings of the National Academy of Science of the United States of America 86, 3194-3198.

Escaffit, F., Perreault, N., Jean, D., Francoeur, C., Herring, E., Rancourt, C., Rivard, N., Vachon, P.H., Paré, F., Boucher, M.P., Auclair, J., Beaulieu, J.F., 2005 Repressed E-cadherin expression in the lower crypt of human small intestine: a cell marker of functional relevance. Experimental Cell Research $302,206-220$

Evans, G.S., Flint, N., Potten, C.S., 1994. Primary cultures for studies of cell regulation and physiology in intestinal epithelium. Annual Review of Physiology 56, 399417.

Föllmann, W., Weber, S., Birkner, S., 2000. Primary cell cultures of bovine colon epithelium: isolation and cell culture of colonocytes. Toxicology In Vitro 14, 435-445.

Halleux, C., Schneider, Y.J., 1991. Iron absorption by intestinal epithelial cells: 1. Caco-2 cells cultivated in serum-free medium on polyethyleneterephtalate microporous membranes, as an in vitro model. In Vitro Cellular and Developmental Biology 27A, 293-302.

Hashim, A., Clyne, M., Mulcahy, G., Akiyoshi, D., Chalmers, R., Bourke, B., 2004. Host cell tropism underlies species restriction of human and bovine Cryptosporidium parvum genotypes. Infection and Immunity 72, 6125-6131.

Holland, R.E., 1990. Some infectious causes of diarrhea in young animals. Clinical Microbiology Reviews 3, 345-375.

Hosoya, K., Tomi, M., Takayama, M., Komokata, Y., Nakai, D., Tokui, T., Nishimura, K., Ueda, M., Obinata, M., Hori, S., Ohtsuki, S., Amidon, G.S., Terasaki, T., 2004 Transporter mRNA expression in a conditionally immortalized rat small intestine epithelial cell line (TR-SIE). Drug Metabolism and Pharmacokinetics 19, 264-269.

Hunt, R.C., Davis, A.A., 1990. Altered expression of keratin and vimentin in human retinal pigment epithelial cells in vivo and in vitro. Journal of Cellular Physiology 145, 187-199.

Jat, P.S., Noble, M.D., Ataliotis, P., Tanaka, Y., Yannoutsos, N., Larsen, L., Kioussis, D., 1991. Direct derivation of conditionally immortal cell lines from an $\mathrm{H}-2 \mathrm{~Kb}$ tsA58 transgenic mouse. Proceedings of the National Academy of Science of the United States of America 88, 5096-5100.
Jha, K.K., Banga, S., Palejwala, V., Ozer, H.L., 1998. SV40-mediated immortalization. Experimental Cell Research 245, 1-7.

Kaeffer, B., Bottreau, E., Velge, P., Pardon, P., 1993. Epitheloid and fibroblastic cell lines derived from the ileum of an adult histocompatible miniature boar $(\mathrm{d} / \mathrm{d}$ haplotype) and immortalized by SV40 plasmid. European Journal of Cell Biology 62, 152-162.

Keller, M.S., Ezaki, T., Guo, R.J., Lynch, J.P., 2004. Cdx1 or Cdx2 expression activates E-cadherin-mediated cell-cell adhesion and compaction in human COLO 205 cells. American Journal of Physiology: Gastrointestinal and Liver Physiology 287, G104-114.

Kerneis, S., Bogdanova, A., Kraehenbuhl, J.P., Pringault, E., 1997. Conversion by Peyer's patch lymphocytes of human enterocytes into $M$ cells that transport bacteria. Science 277, 949-952.

Kuhlmann, I., 1996. The prophylactic use of antibiotics in cell culture. Cytotechnology 19, 95-105.

Lebenthal, A., Lebenthal, E., 1999. The ontogeny of the small intestinal epithelium. Journal of Parenteral and Enteral Nutrition 5, S3-6.

Lelouard, H., Reggio, H., Mangeat, P., Neutra, M., Montcourrier, P., 1999. Mucinrelated epitopes distinguish $\mathrm{M}$ cells and enterocytes in rabbit appendix and Peyer's patches. Infection and Immunity 67, 357-367.

Levy, P., Robin, H., Bertrand, F., Kornprobst, M., Capeau, J., 2003. Butyrate-treated colonic Caco-2 cells exhibit defective integrin mediated signalling together with increased apoptosis and differentiation. Journal of Cellular Physiology 197, 336-347.

Macartney, K.K., Baumgart, D.C., Carding, S.R., Brubaker, J.O., Offit, P.A., 2000. Primary murine small intestinal epithelial cells, maintained in long-term culture, are susceptible to rotavirus infection. Journal of Virology 74, 55975603.

Mayer, L., 2003. Mucosal immunity. Pediatrics 111, 1595-1600.

McCormick, B.A., 2003. The use of transepithelial models to examine host-pathogen interactions. Current Opinion in Microbiology 6, 77-81.

Moll, R., Franke, W.W., Schiller, D.L., Geiger, B., Krepler, R., 1982. The catalog of human cytokeratins: patterns of expression in normal epithelia, tumors and cultured cells. Cell 31, 11-24.

Mundy, R., Schüller, S., Girard, F., Fairbrother, J.M., Phillips, A.D., Frankel, G., 2007. Functional studies of intimin in vivo and ex vivo: implications for host specificity and tissue tropism. Microbiology 153, 959-967.

Murakami, H., Masui, H., 1980. Hormonal control of human colon carcinoma cell growth in serum-free medium. Proceedings of the National Academy of Science of the United States of America 77, 346-348

O'Hare, M.J., Bond, J., Clarke, C., Tacheuki, Y., Atherton, A.J., Berry, C., Moody, J., Silver, A.R.J., Davies, D.C., Alsop, A.E., Neville, A.M., Jat, P.S., 2001. Conditional immortalization of freshly isolated human mammary fibroblasts and endothelial cells. Proceedings of the National Academy of Science of the United States of America 98, 646-651.

Onori, P., Franchitto, A., Sferra, R., Vetuschi, A., Gaudio, E., 2001. Peyer's patches epithelium in the rat: a morphological, immunohistochemical and morphometrical study. Digestive Diseases and Sciences 46, 1095-1104.

Peignon, G., Thenet, S., Schreider, C., Fouquet, S., Ribeiro, A., Dussaulx, E., Chambaz, J., Cardot, P., Pinçon-Raymond, M., Le Beyec, J., 2006. E-cadherin-dependent transcriptional control of apolipoprotein A-IV gene expression in intestinal epithelial cells: a role for the hepatic nuclear factor 4. Journal of Biological Chemistry 281, 3560-3568.

Pryde, S.E., Duncan, S.H., Hold, G.L., Stewart, C.S., Flint, H.J., 2002. The microbiology of butyrate formation in the human colon. FEMS Microbiology Letters 217, 133139.

Qualtrough, D., Hinoi, T., Fearon, E., Paraskva, C., 2002. Expression of CDX2 in normal and neoplastic human colon tissue and during differentiation on an in vitro model system. Gut 51, 184-190.

Quaroni, A., Wands, J., Trelstad, R.L., Isselbacher, K.J., 1979. Epithelioid cell cultures from rat small intestine. Characterization by morphologic and immunologic criteria. The Journal of Cell Biology 80, 248-265.

Quaroni, A., Beaulieu, J.F., 1997. Cell dynamics and differentiation of conditionally immortalized human intestinal epithelial cells. Gastroenterology 113, 11981213.

Rousset, M., Chevalier, G., Rousset, J.P., Dussaulx, E., Zweibaum, A., 1979. Presence and cell growth-related variations of glycogen in human colorectal adenocarcinoma cell lines in culture. Cancer Research 39, 531-534

Rusu, D., Loret, S., Peulen, O., Mainil, J., Dandrifosse, G., 2005. Immunochemical, biomolecular and biochemical characterization of bovine epithelial intestinal primocultures. BMC Cell Biology 6, 42-53.

Santos, R.L., Baümer, A.J., 2004. Cell tropism of Salmonella enterica. International Journal of Medical Microbiology 294, 225-233.

Sauvaget, D., Chauffeton, V., Citadelle, D., Chatelet, F.P., Cywiner-Golenzer, C., Chambaz, J., Pinçon-Raymond, M., Cardot, P., Le Beyec, J., Ribeiro, A., 2002. Restriction of apolipoprotein A-IV gene expression to the intestine villus depends on a hormone-responsive element and parallels differential expression of the hepatic nuclear factor 4 alpha and gamma isoforms. Journal of Biological Chemistry 277, 34540-34548.

Schuller, S., Chong, Y., Lewin, J., Kenny, B., Frankel, G., Phillips, A.D., 2007. Tir phosphorylation and Nck/N-WASP recruitment by enteropathogenic and enterohaemorrhagic Escherichia coli during ex vivo colonization of human intestinal mucosa is different to cell culture models. Cellular Microbiology 9, $1352-1354$

Stamps, A.C., Davies, S.C., Burman, J., O'Hare, M.J., 1994. Analysis of proviral integration in human mammary epithelial cell lines immortalized by retroviral 
infection with a temperature-sensitive SV40 T-antigen construct. International Journal of Cancer 57, 865-874.

Tabuchi, Y., Ohta, S., Arai, Y., Kawahara, M., Ishibashi, K., Sugiyama, N., Horiuchi, T., Furusawa, M., Obinata, M., Fuse, H., Takeguchi, N., Asano, S., 2000. Establishment and characterization of a colonic epithelial cell line MCE301 from transgenic mice harboring temperature-sensitive simian virus 40 large Tantigen gene. Cell Structure and Function 25, 297-307.

Tabuchi, Y., Arai, Y., Ohta, S., Shioya, H., Takahashi, R., Ueda, M., Takeguchi, N., Asano, S., Obinata, M., 2002. Development and characterization of conditionally immortalized gastric epithelial cell lines from transgenic rats harboring temperature-sensitive simian virus 40 large T-antigen gene. Cell Structure Function 27, 71-79.

Tavelin, S., Milovic, V., Ocklind, G., Olsson, S., Artursson, P., 1999. A conditionally immortalized epithelial cell line for studies of intestinal drug transport. The Journal of Pharmacology and Experimental Therapeutics 290, $1212-1221$.
Toouli, C.D., Huschtscha, L.I., Neumann, A.A., Noble, N.R., Colgin, L.M., Hukku, B. Reddel, R.R., 2002. Comparison of human mammary epithelial cells immortalized by simian virus $40 \mathrm{~T}$-Antigen or by the telomerase catalytic subunit. Oncogene 21, 128-139.

Velge, P., Bottreau, E., Quéré, P., Pardon, P., Nicolle, J.C., Morisson, M., Bout, D. Dimier, I., 2002. Establishment and characterization of partially differentiated chicken enterocyte cell clones. European Journal of Cell Biology 81, 203 212.

Wice, B.M., Trugnan, G., Pinto, M., Rousset, M., Chevalier, G., Dussaulx, E., Lacroix, B. Zweibaum, A., 1985. The intracellular accumulation of UDP-N-acetylhexosamines is concomitant with the inability of human colon cancer cells to differentiate. Journal of Biological Chemistry 260, 139-146.

Zweibaum, A., Pinto, M., Chevalier, G., Dussaulx, E., Triadon, N., Lacroix, B., Haffen, K., 1985. Enterolytic differentiation of a subpopulation of the human colon tumor cell line HT-29 selected for growth in sugar-free medium and its inhibition by glucose. Journal of Cellular Physiology 122, 21-29. 